\title{
Genetic associations between Johne's disease and susceptibility to Mycobacterium bovis and Mycobacterium avium subsp avium in Irish Holstein Friesian dairy cows
}

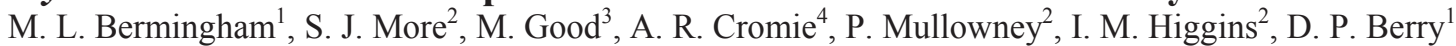

${ }^{1}$ Moorepark Production Research Centre, Fermoy, Co. Cork, Ireland, ${ }^{2}$ Centre for Veterinary Epidemiology and Risk Analysis, UCD School of Agriculture, Food Science and Veterinary Medicine, University College Dublin, Belfield, Dublin 4, Ireland, ${ }^{3}$ Department of Agriculture, Fisheries and Food, Kildare St, Dublin 2, Ireland, ${ }^{4}$ The Irish Cattle Breeding Federation, Bandon, Co. Cork, Ireland

Email: mbermingham@hotmail.com

Introduction Johne's disease in cattle is caused by Mycobacterium avium subsp paratuberculosis. The indications are that the prevalence of Johne's disease has increased in Ireland since the introduction of the single European market (Good et al. 2009). A recent study has demonstrated that significant genetic variation exists for susceptibility to M. a. paratuberculosis infection in Irish Holstein Friesian dairy cows, indicating that genetic improvement is possible (Berry et al. 2009). Nevertheless, data on Johne's disease occurrence is not collected routinely on Irish dairy farms. The shared evolutionary history of, and similar immunopathological response post-infection to, Mycobacterium bovis and Mycobacterium avium subsp avium may allow them to be used as indicators of susceptibility to $M$. a. paratuberculosis infection in cattle. National single intradermal comparative tuberculin test data collected routinely as part of the Irish bovine tuberculosis eradication programme may therefore provide a means of indirect selection for Johne's disease resistance in Irish dairy cattle. The objective of this study was to estimate the genetic associations between resistance to M. a. paratuberculosis infection and measures of susceptibility to M. bovis and M. a. avium infection.

Material and methods Serological response to M. a. paratuberculosis was used as a measure of cow susceptibility to Johne's disease. Data on animal serological response to $M$. a. paratuberculosis were obtained from 44 Irish herds from the Irish Department of Agriculture, Fisheries and Food between June 2004 and November 2005. Blood samples were collected from all lactating and non-lactating animals older than 12 months, and M. a. paratuberculosis specific enzyme linked immunosorbent assay (ELISA) was conducted. Serological response to M. a. paratuberculosis was dichotomised as seropositive (sample to positive control optical density ratio greater or equal to 70) or seronegative (sample to positive control optical density ratio less than 70). Only cows with a known sire, and herds with at least two ELISA positive cows (one of which was home bred) and 10 or more tested cows were retained. The final dataset included in the analysis of serological response to M. a. paratuberculosis consisted of 4,581 cows from 38 herds. The single intradermal comparative tuberculin test (SICTT) was used as a measure of susceptibility of cows to M. bovis and M. a. avium infection. The test involves injecting $M$. bovis-purified protein derivative (PPD) into the neck of each animal, and comparing the reaction induced to that produced by $M$. avium-PPD (a measure of sensitisation to environmental mycobacteria). Susceptibility to $M$. bovis-PPD responsiveness was dichotomised as standard reactor (a $M$. bovis-PPD reaction $4 \mathrm{~mm}$ or greater than the $M$. avium-PPD reaction) or nonreactor (a $M$. bovis-PPD reaction equal to the $M$. avium-PPD reaction). Susceptibility to $M$. avium-PPD responsiveness was dichotomised as standard reactor (a $M$. avium -PPD reaction $4 \mathrm{~mm}$ or greater than the $M$. bovis-PPD reaction) or nonreactor. National SICTT records between November 2000 and December 2007 were available for inclusion in the analysis. An episode was defined as a herd restriction initiated by two or more standard reactors (with at least one of the animals being home bred), and terminated by 2 consecutive clear herd tests. Cows that calved outside the normal age for a given parity, that had inconclusive SICTT results, or that moved into the herd within six weeks of the SICTT (it takes three to six weeks to develop a positive reaction to the test post infection) were discarded. Following edits, only episodes with at least one standard reactor and ten or more tested cows were retained; 19,663 M. bovis-PPD responsiveness records from 640 episodes and 15,824 M. avium-PPD responsiveness records from 479 episodes remained. Genetic and residual (co)variance components between serological response to $M$. a. paratuberculosis and susceptibility to M. bovis-PPD and $M$. avium-PPD responsiveness were estimated using bivariate linear animal models in ASREML (Gilmour et al. 2009). The likelihood ratio test of nested models was used to determine whether the genetic correlations differed significantly from zero.

Results Heritability estimates for serological response to $M$. a. paratuberculosis, susceptibility to $M$. bovis-PPD responsiveness and susceptibility to $M$. avium-PPD responsiveness were 0.07 (standard error [SE] $=0.03$ ), 0.03 ( $\mathrm{SE}=0.01$ ) and $0.03(\mathrm{SE}=0.01)$ respectively. A weak non significant genetic correlation of $0.03(\mathrm{SE}=0.32 ; \mathrm{P}>0.05)$ was estimated between serological response to $M$. a. paratuberculosis and susceptibility to M. bovis-PPD responsiveness. However, a strong positive genetically correlation of $0.84(\mathrm{SE}=0.20 ; \mathrm{P}<0.05)$ was estimated between serological response to $M$. $a$. paratuberculosis and susceptibility to $M$. avium-PPD responsiveness

Conclusions The results from this study suggest that selection for reduced M. avium-PPD responsiveness may indirectly increase resistance to $M$. a. paratuberculosis infection. $M$. avium-PPD responsiveness data is collected routinely within the national bovine tuberculosis eradication program; therefore, it should be possible to develop breeding programs to select for increased resistance to Johne's disease, via the SICTT. However, data in this study were few and results should therefore be interpreted with caution.

Acknowledgements Financial support from ERAD and Stimulus Research Fund (RSF-060328) is gratefully acknowledged

\section{References}

Berry, D.P., Good, M., Mullowney, P., Cromie, A.R., \& More, S.J. 2009. Journal of Dairy Science. (in press).

Good, M., Clegg, T., Sheridan, H., Yearsely, D., O’Brien, T., Egan, J., \& Mullowney, P. 2009. Ir. Vet. J. 62, $597-606$.

Gilmour, A.R., Cullis, B.R., Welham, S.J., \& Tompson, R. 2009. ASReml reference manual, $2^{\text {nd }}$ edition. 